\title{
Intra-mitochondrial degradation of Tim23 curtails the survival of cells rescued from apoptosis by caspase inhibitors
}

\author{
CG Goemans ${ }^{\star, 1,2}$, P Boya $^{1,3}$, CJ Skirrow ${ }^{1}$ and AM Tolkovsky ${ }^{\star, 1}$
}

Caspase inhibition can extend the survival of cells undergoing apoptosis beyond the point of mitochondrial outer membrane permeabilisation (MOMP), but this does not confer long-term protection because caspase-independent death pathways emerge. Here, we describe a novel mechanism of mitochondrial self-destruction in caspase-inhibited cells, whose hallmark is the degradation of Tim23, the essential pore-forming component of the TIM23 inner membrane translocase. We show that Tim23 degradation occurs in cycling and post-mitotic cells, it is caspase-independent but Bax/Bak dependent, and it follows cytochrome $c$ release. The proteolytic degradation of Tim23 is induced by MOMP and is mitochondrion-autonomous, as it also occurs in isolated mitochondria undergoing permeability transition. Degradation of Tim23 is selective, as expression of several other inner membrane proteins that regulate respiratory chain function is unaffected, and is not autophagic, as it occurs similarly in autophagy-proficient and -deficient (Atg-5 knockout) cells. Depleting Tim23 with siRNA is sufficient to inhibit cell proliferation and prevent long-term survival, while expression of degradation-resistant Tim23-GFP in mitochondria delays caspaseindependent cell death. Thus, mitochondrial autodigestion of Tim23 joins the array of processes contributing to caspaseindependent cell death. Because mitochondrial biogenesis requires a functional protein-import machinery, preventing Tim23 degradation might, therefore, be essential for repairing damaged mitochondria in chronic degenerative diseases.

Cell Death and Differentiation (2008) 15, 545-554; doi:10.1038/sj.cdd.4402290; published online 4 January 2008

Most mitochondrial proteins, apart from 13 polypeptides, are synthesised in the cytoplasm of eukaryotic cells. Mitochondrion-targeted proteins enter the organelle by import through a multiprotein translocation complex in the outer membrane (TOM). Two different inner-membrane translocation complexes then shuttle proteins to further destinations: TIM22 mediates the assembly of innermembrane proteins while TIM23 mostly imports proteins into the matrix. ${ }^{1}$

Apart from providing cellular ATP, mitochondria are also integral components of proapoptotic signalling. During apoptosis, mitochondrial outer membrane permeabilisation (MOMP) causes release of apoptogenic factors such as cytochrome $c$ from the intermembrane space (IMS) to the cytoplasm. Cytochrome c-mediated activation of caspases via Apaf- 1 is a dominant regulator of apoptosis. ${ }^{2}$ Although pan-specific caspase inhibitors can extend survival considerably depending on the cell type and death stimulus, caspaseindependent mechanisms of death execution take over in the long term. This has been ascribed to the action of other mitochondrial apoptogenic factors, such as AIF or EndoG. ${ }^{2}$ In addition, autophagy, which is co-activated by many apoptotic stimuli, has pro-death activity when apoptosis execution is impaired. $^{3,4}$
Exploiting mechanisms that suppress mitochondrial dysfunction and its deleterious follow-on effects is an obvious starting point for the development of treatments against loss of irreplaceable cells during neurodegenerative diseases, stroke, or myocardial infarction. However, therapeutic strategies should also address ways to mend defective mitochondria, thereby hopefully achieving long-term protection. Therefore, it is important to assess whether damaged mitochondria retain a functional import machinery so that the supply of cytoplasmic proteins is maintained.

Here we focus on Tim23, the eponymous constituent of the TIM23 complex that forms the crucial pore-forming innermembrane translocase. ${ }^{5}$ We show that cells induced to undergo apoptosis in the presence of caspase inhibitors selectively degrade Tim23. Degradation is independent of macroautophagy, requires Bax/Bak and is mitochondrionautonomous. Given that depletion of endogenous Tim23 by RNAi precludes long-term survival of otherwise untreated cells and a stabilised Tim23 delays caspase-independent cell death, our results suggest that apoptosis-induced Tim23 degradation contributes to caspase-independent cell death. Hence, although cytoplasmic messengers may initiate proapoptotic events on mitochondria, intra-mitochondrial proteases, by eliminating a key protein import pathway, uncouple

\footnotetext{
${ }^{1}$ Department of Biochemistry, University of Cambridge, Cambridge, UK

*Corresponding authors: CG Goemans, AM Tolkovsky, Department of Biochemistry, University of Cambridge, Tennis Court Road, Cambridge CB2 1QW, UK. Tel: + 441223 339319; Fax: + 441223 333345; E-mails: christoph.goemans@ @ub.de or amt@mole.bio.cam.ac.uk

${ }^{2}$ Current address: Molecular Neurobiochemistry, Ruhr-University Bochum, D-44780 Bochum, Germany

${ }^{3}$ Current address: 3D Lab, Department of Cellular and Molecular Physiopathology, Centro de Investigaciones Biológicas, CSIC, E-28040 Madrid, Spain Keywords: caspase-independent cell death; Bax/Bak; TIM23 translocase; proteolysis; mitochondria

Abbreviations: AraC, cytosine arabinoside; Atr, atractyloside; BAF, boc-aspartyl(OMe)-fmk; BrdU, 5-bromo 2'-deoxyuridine; CHX, cycloheximide; CsA, cyclosporine A; IEto, etoposide; F1 $\beta$, ATPsynthase $\beta$; MOMP, mitochondrial outer membrane permeabilisation; Sts, staurosporine

Received 21.5.07; revised 24.10.07; accepted 12.11.07; Edited by RJ Youle; published online 04.1.08
} 
mitochondria from the symbiotic relationship with their host, causing inevitable cellular demise.

\section{Results}

Apoptotic inducers stimulate selective Tim23 degradation. HeLa cells were treated with various apoptotic inducers in the absence or presence of the pan-caspase inhibitor boc-aspartyl(OMe)-FMK (BAF) (Figure 1a). In the presence of BAF, treatment with staurosporine (Sts), etoposide (Eto), UV irradiation, or expression of Puma, but not mutant Puma lacking the $\mathrm{BH} 3$ domain (Puma $\Delta \mathrm{BH} 3$ ), diminished the amount of Tim23 by $60-90 \%$ within $1-2$ days, but the amount of $\mathrm{Hsp} 60$ (a mitochondrial matrix protein) or ATP synthase $\beta(\mathrm{F} 1 \beta$, an inner-membrane-associated protein) did not change. Similar results were obtained using Z-VAD-FMK (Supplementary Figure S1a) or the viral caspase inhibitor p35 (Supplementary Figure S1b). All the stimuli that caused Tim23 degradation also induced $60-100 \%$ apoptosis within $24 \mathrm{~h}$, which was effectively suppressed by BAF (Figure 1b). Hence, Tim23 degradation is not mediated by caspases. Degradation of Tim23 was observed only in those cells that were kept alive with BAF and not in cells that die in the absence of caspase inhibitors (Supplementary Figure S1c), indicating that Tim23 degradation is not an essential part of the apoptotic programme.

To investigate whether the response was specific to cellcycle proficient human cells, expression of Tim23 was examined in post-mitotic rat sympathetic ganglion (SCG) neurons. Neurons were stimulated to undergo apoptosis in the presence of BAF by withdrawal of nerve growth factor (NGF) or by treatment with cytosine arabinoside (AraC) in the presence of NGF. These two stimuli use alternative signalling

a HeLa
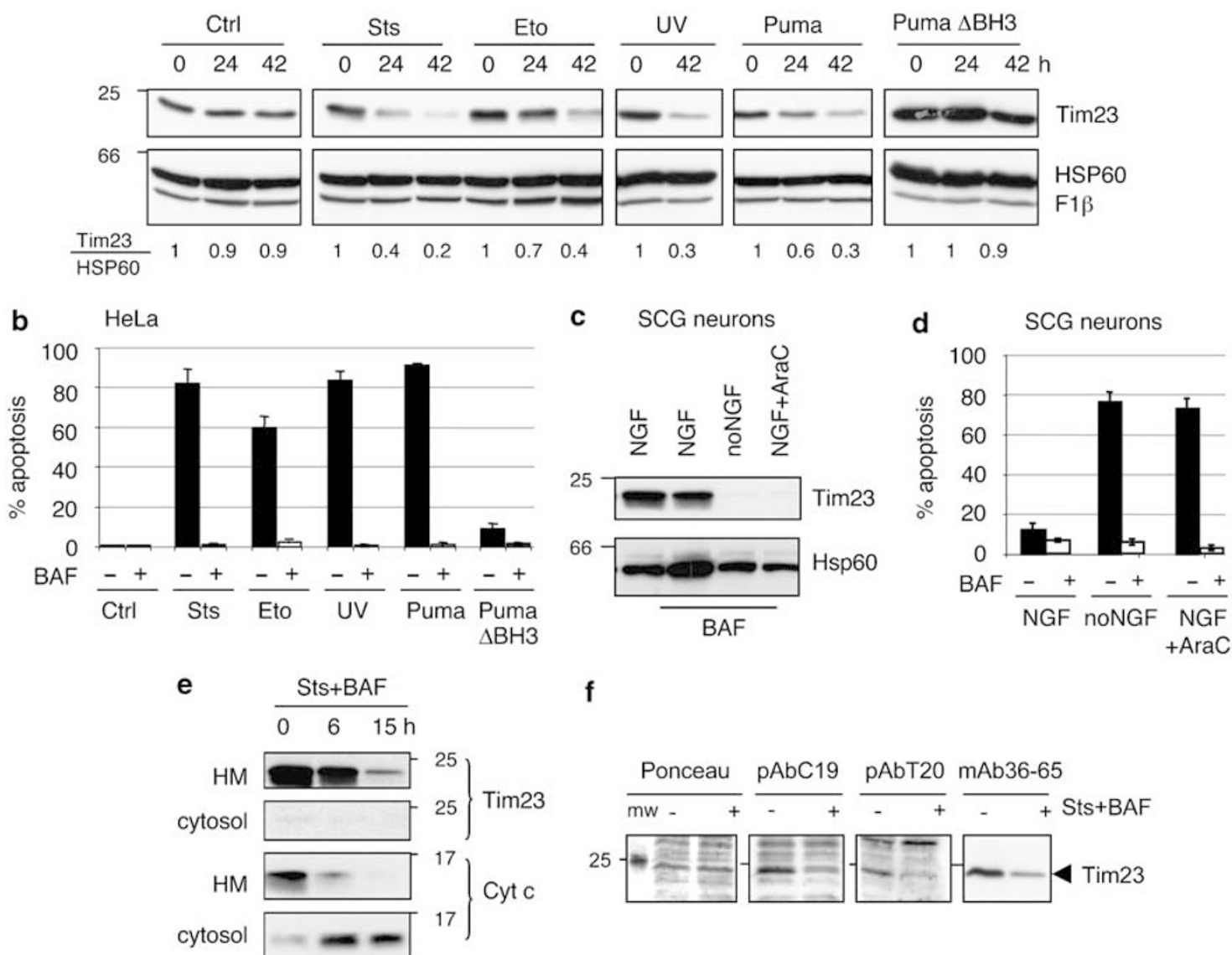

f

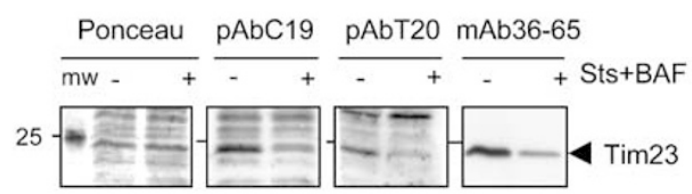

Figure 1 Tim23 is selectively degraded upon induction of apoptosis when death is delayed by caspase inhibition. (a) HeLa cells were induced to undergo apoptosis with $1 \mu \mathrm{M}$ staurosporine (Sts), $100 \mu \mathrm{M}$ etoposide (Eto), UVC irradiation $\left(3 \mu \mathrm{J} / \mathrm{cm}^{2}\right.$ ), Ad-hPuma (Puma) or Ad-hPuma $\Delta \mathrm{BH} 3$ (Puma $\Delta \mathrm{BH} 3$ ) in the presence of $100 \mu \mathrm{M} \mathrm{BAF}$. At 24 and $42 \mathrm{~h}$, cell lysates were analysed by immunoblotting with antibodies against Tim23, Hsp60 or ATP synthase $\beta(\mathrm{F} 1 \beta)$. Numbers below the blot show ratio of band intensity for Tim23/Hsp60. (b) HeLa cells treated as in (a) but in the absence or presence of BAF were scored for apoptosis after $24 \mathrm{~h}$. Mean \pm S.D., three independent experiments in duplicate wells. (c) Rat superior cervical ganglion (SCG) neurons cultured as indicated ( $50 \mathrm{ng} / \mathrm{ml}$ NGF, $1 \mathrm{mM} \mathrm{AraC,} 100 \mu \mathrm{M} \mathrm{BAF}$ ) were lysed after 2 days and analysed by immunoblotting for Tim23 and Hsp60. (d) SCG neurons were scored for apoptosis after $17 \mathrm{~h}$ (mean \pm range, two independent experiments in triplicate wells). (e) HeLa cells treated with Sts and BAF (as in a) were fractionated into a mitochondrial pellet (heavy membranes, $\mathrm{HM}$ ) and supernatant (cytosol) and lysates probed with antibodies against Tim23 or cytochrome $c$ (cyt c). (f) HeLa cells were treated for $24 \mathrm{~h}$ with $1 \mu \mathrm{M}$ Sts and $100 \mu \mathrm{M}$ BAF or left untreated. Equal amounts of the two lysates were loaded in pairs onto the same gel three times. A pair of lanes were analysed by immunoblotting using either an anti-Tim23 polyclonal antibody directed against an epitope at the C-terminus (pAbC19), or near the C-terminus (pAbT20), or the monoclonal anti-Tim23 antibody that detects an epitope between amino acids 36-65 (mAb36-65). Ponceau S staining of one of the pairs shows equal protein loading. Molecular mass markers are shown on the left; the arrow indicates the position of Tim23 
mechanisms that converge on activation of Bax. ${ }^{6,7}$ As in the HeLa cells, the amount of Tim23 also decreased in BAFtreated neurons within 1 day during apoptosis induction (Figure 1c), where death was completely suppressed by BAF (Figure 1d) for several days, as reported previously. ${ }^{8}$ Thus, loss of Tim23 is of general significance as it is induced in dividing or post-mitotic cells by several types of apoptotic stimuli (Sts, DNA damage, or growth factor withdrawal).

To investigate whether cytochrome $c$ release from mitochondria into the cytosol preceded degradation of Tim23, HeLa cells treated with Sts and BAF were fractionated into a mitochondrial heavy membrane fraction $(\mathrm{HM})$ and remaining cytosol at different times after treatment onset. By $6 \mathrm{~h}$, mitochondrial cytochrome $c$ was almost completely released into the cytosol but there was only $\sim 15 \%$ decrease in Tim23. However, by $15 \mathrm{~h}$, Tim23 degradation was nearly complete (Figure 1e). Hence, Tim23 degradation follows the release of cytochrome $c$, implying that it occurs in consequence to MOMP.

The anti-Tim23 antibody used is a monoclonal antibody raised against amino acids 5-126 (the N-terminal half of the molecule). In yeast, the first 20 amino acids of Tim23 protrude through the outer membrane ${ }^{9}$ and are cleavable by exogenously added proteases, but in vertebrates this topology is unlikely because the $\mathrm{N}$-terminus is shorter by 21 amino acids, the missing amino acids encompassing the cleavage region. ${ }^{10}$ However, to ensure that loss of Tim23 immunoreactvity was not due to clipping off of the very $\mathrm{N}$-terminus leaving behind a protein that may still function as a pore, we mapped the recognition epitope. Transfection of HeLa cells with constructs encoding a series of deletions in the $\mathrm{N}$ terminal half of Tim23-EGFP, and analysis of the expressed proteins with anti-Tim23 or anti-GFP, showed that the epitope was located between amino acids 36 and 65 (Supplementary Figure S2a and b). Thus, loss of Tim23 immunoreactivity was not simply due to deletion of the first few amino acids. Moreover, Tim23 degradation was also observed using two polyclonal antibodies (Figure 1f) raised against C-terminal peptide epitopes that are at (pAbC19) or close to (pAbT20) the $\mathrm{C}$-terminus. These epitopes encompass the matrix-projecting 10-amino-acid C-terminus and the fourth transmembrane domain of Tim23. ${ }^{10}$ These observations suggest that Tim23 degradation envelops the whole molecule.

Tim23 degradation depends on Bax/Bak and is not autophagic. Bax and Bak are crucial mediators of apoptotic MOMP, and lack of both prevents mitochondrion-mediated apoptosis. ${ }^{11}$ To determine whether Tim23 degradation is the result of Bax/Bak activities, Sts and BAF were added to mouse embryonic fibroblasts (MEFs) derived from wild-type (wt), singly-deficient Bax-KO or Bak-KO, or Bax/Bak double knock out (DKO) mice. There was $50-80 \%$ loss of Tim23 in wt, Bax KO, or Bak KO MEFs after $24 \mathrm{~h}$, but in DKO MEFs there was complete protection against Sts-induced Tim23 degradation (Figure 2a). Likewise, DKO MEFs maintained Tim23 when Eto or Puma (Supplementary Figure S1c and d) was used as apoptotic stimuli.

$\mathrm{We}^{3}$ and others ${ }^{4,12}$ have suggested that damaged mitochondria are slowly eliminated by macroautophagy (referred to here as autophagy) in apoptotic cells in which caspases are
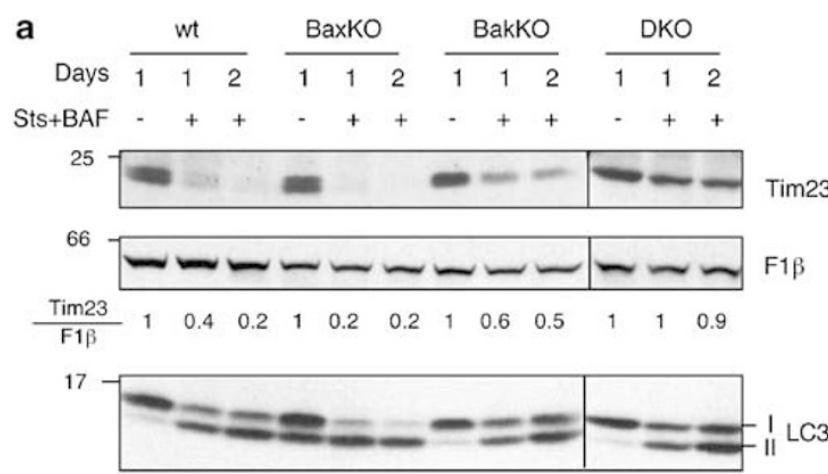

b

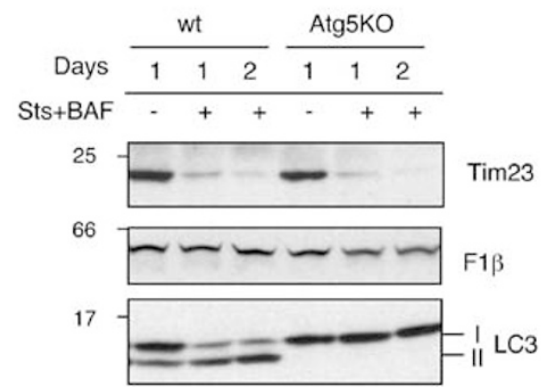

Figure 2 Degradation of Tim23 is dependent on Bax/Bak and is not mediated by macroautophagy. (a) Wild type (wt), Bax-KO, Bak-KO or Bax/Bak double KO (DKO) MEFs treated with $1 \mu \mathrm{M}$ Sts and $100 \mu \mathrm{M} \mathrm{BAF}$ for 1 or 2 days were lysed and analysed by immunoblotting for Tim23 and F1 $\beta$.Numbers show ratio of band intensities for Tim23/F1 $\beta$. Expression of the autophagy marker LC3 in its soluble (LC3-I) and autophagosome-associated form (LC3-II); increased LC3-II indicates that autophagy has been induced. (b) MEFs derived from matched wt (different from the line in a) and Atg5-KO macroautophagy-deficient mice were treated as in (a). Note lack of LC3-II in Atg5-KO MEFs. Results are representative of three independent experiments

inhibited. We tested whether Tim23 degradation is mediated by autophagy by examining whether it is degraded in DKO cells, where autophagy is activated by apoptotic stimuli, ${ }^{13}$ and also by stimulating apoptosis in MEFs lacking Atg5 (Atg5-KO), a key regulator of autophagosome formation in eukaryotic cells. ${ }^{14}$ Autophagic activity was detected by measuring the conversion of MAP-LC3 (referred to here as LC3) from a soluble form (LC3-I) to an autophagosome-associated form (LC3-II). LC3-II formation occurs due to lipid modification of LC3-I after a series of priming events mediated by Atg5 in conjunction with other Atg genes. ${ }^{15,16}$ Figure 2a shows that conversion of LC3-I to LC3-II occurred equally well in all four MEF lines treated with Sts and BAF, including DKO, as reported previously. ${ }^{13}$ Moreover, Sts caused Tim23 degradation in autophagy-deficient Atg5-KO MEFs (Figure 2b). As shown in Figure 1 (and Figure 4), we also noted that other mitochondrial proteins did not disappear simultaneously with Tim23, as might be expected if a significant number of entire mitochondria were digested in lysosomes. Thus, the relatively rapid Tim23 degradation induced by apoptotic stimuli is Bax/ Bak-dependent but autophagy-independent.

Tim23 degradation is mitochondrion-autonomous. After MOMP, the inner mitochondrial membrane can become accessible to cytoplasmic proteases. The rapid loss of mitochondrial membrane potential $(\Delta \Psi \mathrm{m})$ and the 


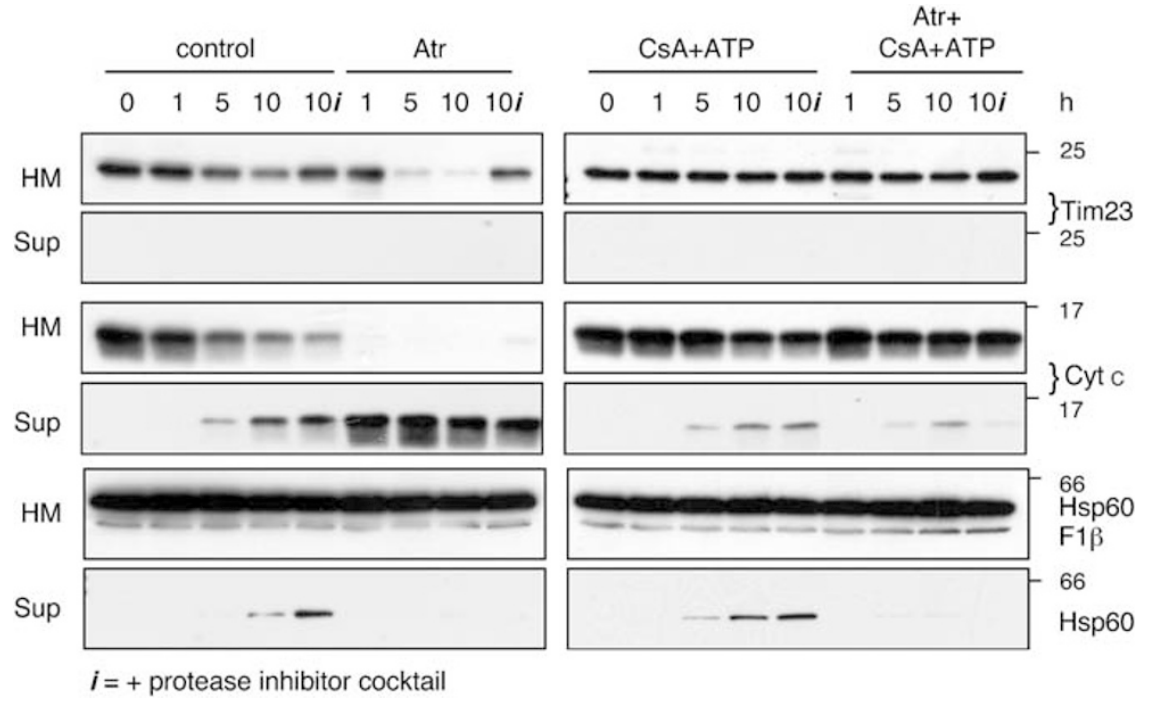

Figure 3 Tim23 is degraded by intra-mitochondrial proteases. Rat liver mitochondria $(0.3-0.5 \mathrm{mg})$ were suspended in $100 \mu \mathrm{l}$ of K/MOPS buffer without or with $1 \mathrm{mM}$ ATP and $5 \mu \mathrm{M} \mathrm{CsA}$ (cyclosporine A). Atr (5 mM) was added where indicated. One sample of each condition also contained EDTA-free complete ${ }^{\mathrm{TM}}$ protease inhibitors $(i)$. After incubation at $30^{\circ} \mathrm{C}$, mitochondria were pelleted at $10000 \times g$ and resuspended in $100 \mu$ l. Pellets (HM) and supernatants (Sup) (15 $\mu$ each) were analysed for Hsp60, $\mathrm{F} 1 \beta$, Tim23, or cyt $c$ by immunoblotting. Results are representative of three independent experiments

generation of reactive oxygen species (ROS) after induction of apoptosis have been ascribed to caspases entering damaged mitochondria and degrading respiratory or other proteins. ${ }^{17,18}$ To test whether the proteolytic activity that degrades Tim23 resides within the cytoplasm and enters the mitochondria after MOMP, or resides within the mitochondria, we tested whether Tim23 would be degraded in isolated rat liver mitochondria after induction of MOMP. MOMP was induced by treatment with atractyloside (Atr), which binds to the adenine nucleotide transporter and increases the probability of MOMP. ${ }^{19}$ Atr was active as it triggered $\mathrm{Ca}^{2+}$-dependent mitochondrial swelling in the same solution we used to examine Tim23 degradation ${ }^{20}$ (data not shown). Cohort samples were incubated in the presence of cyclosporine A (CsA) and ATP, inhibitors of Atr function. ${ }^{21,22}$ To demonstrate intra-mitochondrial proteolytic activity, treated mitochondria were also incubated in the presence of EDTA-free Complete ${ }^{\mathrm{TM}}$, a protease inhibitor mix that supported $\mathrm{Ca}^{2+}$-dependent MOMP (data not shown).

Figure 3 shows that Atr promoted specific Tim23 degradation within $5 \mathrm{~h}$, after cytochrome $c$ release (at $1 \mathrm{~h}$ ), the same order of events observed in intact cells (Figure 1e) although greatly accelerated. Little release of matrix-localised Hsp60 or inner membrane-associated $\mathrm{F} 1 \beta$ was observed up to $10 \mathrm{~h}$ after Atr addition, indicating that Atr induced selective outermembrane rupture. In the control buffer, Tim23 was largely maintained in the mitochondria up to $10 \mathrm{~h}$ (the small amount of cytochrome $c$ released with Hsp60 representing nonspecific dissolution of mitochondria). Atr-induced Tim23 degradation was inhibited by co-incubation with CsA and ATP, demonstrating that mitochondria treated with Atr underwent selective MOMP. Most notably, addition of a protease inhibitor mix completely prevented Tim23 degradation, although cytochrome $c$ release proceeded unchanged, indicating that MOMP had occurred normally. Thus, MOMP is sufficient to induce proteolytic Tim23 degradation by mechanisms resident within the mitochondria.

Degradation of Tim23 is highly selective. Hsp60 and $\mathrm{F} 1 \beta$ are not integral membrane proteins. To examine the selectivity of Tim23 degradation, we investigated the lifetime of several additional membrane proteins. In HeLa cells treated with Sts or UV irradiation (and BAF) for $24 \mathrm{~h}$, conditions where Tim23 was degraded, there was no loss of Tom20, a key constituent of the TOM complex in the outer membrane ${ }^{5}$ that delivers cargo to Tim23 (Figure 4a). The increased intensity of Tom20 fluorescence is most likely due to mitochondrial clustering that occurs after their fragmentation, ${ }^{23}$ as there was no significant change in Tom20 expression measured by immunoblotting (Figure 4b).

To investigate the lifetime of mitochondrial inner-membrane components of the respiratory chain, we followed proteins encoded by mitochondrial DNA after metabolic labelling of mitochondrial translation products in cells (Figure 4b). ${ }^{24}$ In the presence of emetine (eme), an irreversible inhibitor of cytoplasmic translation, four prominent bands were labelled at approximate masses of $38,32,22$, and $21 \mathrm{kDa}$ (identified as ND2 (complex I), cytochrome $b$ (complex III), COX subunits I-III (complex IV), and A6 (complex V) ${ }^{24}$ ). The same four bands were prominently labelled in the presence of cycloheximide $(\mathrm{CHX})$ (the reversible inhibitor of cytoplasmic translation) although there was more background labelling of cellular translation products. However, labelling of all four bands (but not the background bands) was ablated when $\mathrm{CHX}$ was added together with chloramphenicol (CAP), the selective inhibitor of mitochondrial translation, confirming that these are specific mitochondrial translation products. CAP similarly ablated labelling in the presence of eme (data not shown). After $\mathrm{CHX}$ was washed out and protein synthesis resumed, cells were further incubated in the absence or presence of Sts 
Ctrl

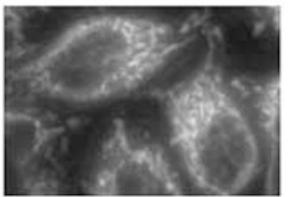

Sts

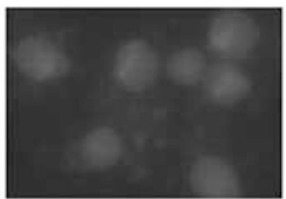

UV

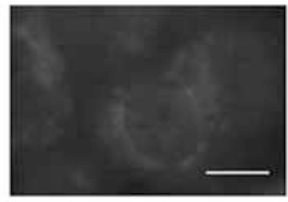

C

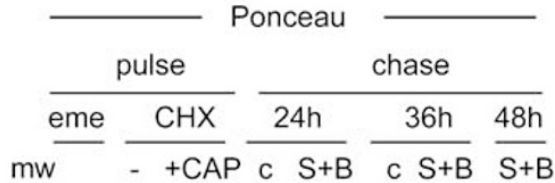

Tom20
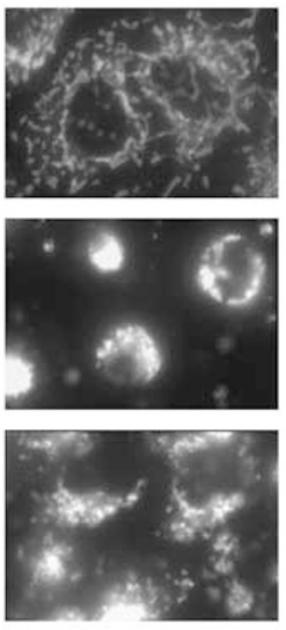

b
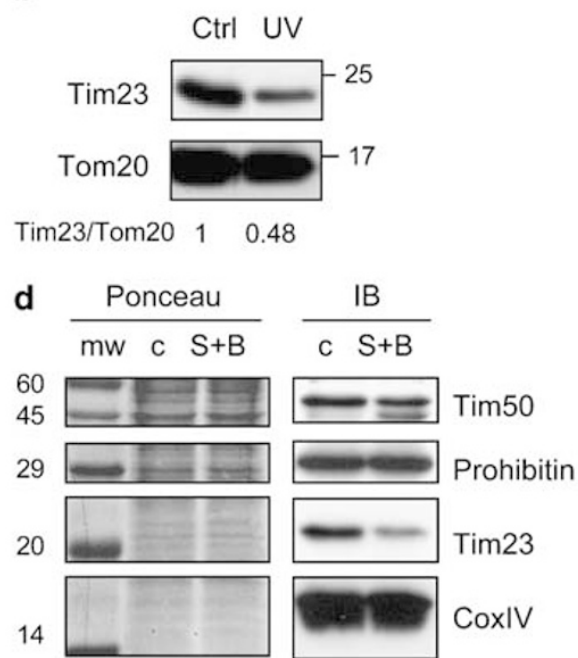

$\left[{ }^{35} \mathrm{~S}\right]$ incorporation

\begin{tabular}{|c|c|c|c|c|}
\hline \multicolumn{2}{|c|}{ pulse } & \multicolumn{3}{|c|}{ chase } \\
\hline eme & $\mathrm{CHX}$ & $24 \mathrm{~h}$ & $36 \mathrm{~h}$ & $48 \mathrm{~h}$ \\
\hline
\end{tabular}

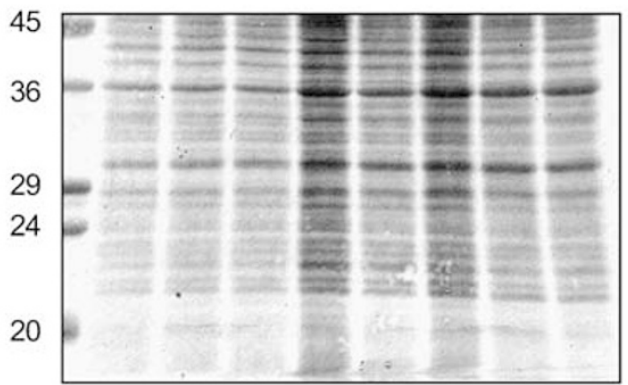

Figure 4 Several mitochondrial integral membrane proteins are preserved during degradation of Tim23. (a) HeLa cells treated with $1 \mu \mathrm{M} \mathrm{Sts} \mathrm{or} 3 \mu \mathrm{J} / \mathrm{cm}^{2} \mathrm{UVC}$ in the presence of $100 \mu \mathrm{M}$ BAF were fixed after $24 \mathrm{~h}$ and stained with anti-Tim23 or anti-Tom20 followed by Alexa 488-conjugated second antibody. Fluorescent images were captured at a fixed exposure. Bar $=10 \mu \mathrm{m}$. (b) Immunoblot of Tim23 and Tom20 from a parallel set of UV-treated cells. Results are representative of three independent experiments. (c) Proteins encoded by mitochondrial DNA were metabolically labelled in HeLa cells with ${ }^{35}$ Slaminoacids as described in Materials and Methods. One cohort was harvested immediately after the labelling to measure the intensity of labelling at the beginning of the chase period (pulse). The other cohorts that had been labelled only in the presence of $\mathrm{CHX}$ were washed free of $\mathrm{CHX}$ (chase) and incubated for 24,36 , or $48 \mathrm{~h}$ in the presence of DMEM and $10 \%$ serum (con), or the same medium containing $1 \mu \mathrm{M}$ Sts and $75 \mu \mathrm{M}$ BAF $(S+B)$. Proteins from the mitochondrial fraction were separated by PAGE, electroblotted, and imaged by exposure to a phosphorimaging screen. Mitochondrial proteins indicated by arrows were identified according to their molecular mass and the labelling pattern described by Chomyn ${ }^{26}(\mathrm{COI}, \mathrm{II}, \mathrm{III}, \mathrm{cytochrome} c \mathrm{coxidase}$ subunits 1, 2, 3; ND2, rotenone-sensitive NADH dehydrogenase subunit 2, Cyt $b$, cytochrome $b$; A6, subunit 6 of the F1Fo ATPase). Left panel: Ponceau S staining; right panel: scanned phosphorimage. Emetine (eme) and chloramphenicol (CAP), specific inhibitors of cytoplasmic and mitochondrial translation, respectively, were used to corroborate mitochondrial translation products (see text for details). Results are representative of four independent experiments. (d) Immunoblot of proteins from cells that had been treated with $\mathrm{CHX}$ and washed exactly as in panel $\mathbf{c}$ but in the absence of radiolabel and post-incubated for $36 \mathrm{~h}$ in the absence or presence of Sts $+\mathrm{BAF}$. Left panel: Ponceau S image; right panel: blot exposed to anti-Tim50, anti-prohibitin, anti-Tim23, and anti-COX IV. Tim23 intensity decreased by $\sim 80 \%$, and that of Tim50 by $\sim 10 \%$ and there was no decrease in the intensity of prohibitin or COX IV after Sts + BAF treatment. Results are representative of three independent experiments

and BAF, to induce Tim23 degradation. Figure $4 \mathrm{c}$ shows that none of the mitochondrion-encoded polypeptides that were selectively labelled in the presence of $\mathrm{CHX}$ were degraded up to $48 \mathrm{~h}$ following the addition of Sts and BAF, although by $36 \mathrm{~h}$ Tim23 detected by immunoblotting was degraded by $80 \%$ under the same conditions (Figure 4d). Ponceau staining shows that there was no loss of proteins generally over the time of the experiment. Moreover, under equivalent conditions, neither prohibitin nor COX IV expression-two additional integral inner-membrane proteins encoded by nuclear DNA-was diminished (Figure 4d). Tim 50, an inner-membrane partner of Tim23 in the import process, ${ }^{25}$ was slightly reduced by $\sim 10 \%$. Thus, among the proteins investigated, Tim23 appears to be an extraordinarily unstable inner-membrane protein following activation of apoptosis in the presence of caspase inhibitors.

Depletion of Tim23 impairs mitochondrial import, inhibits cell cycle progression, and precludes longterm survival. To assess the biological significance of loss of Tim23, we examined whether depletion of Tim23 in HeLa cells in the absence of apoptotic stimulation would impede 
a
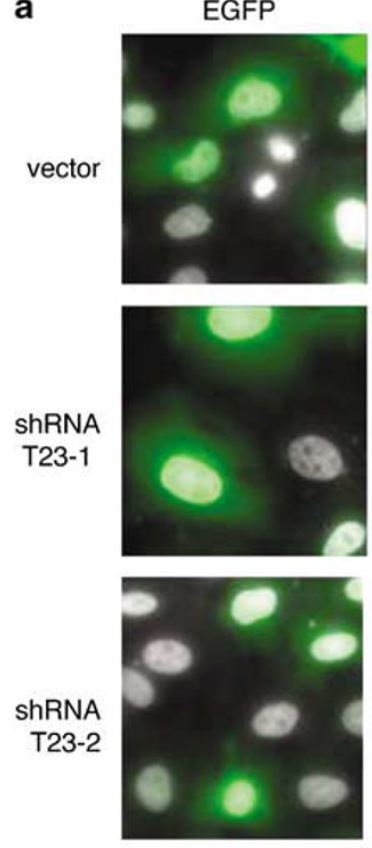

C

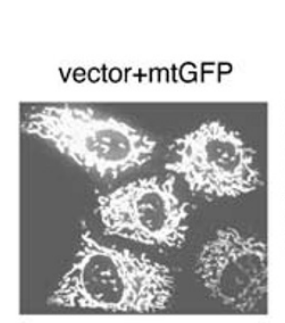

T23-1+mtGFP

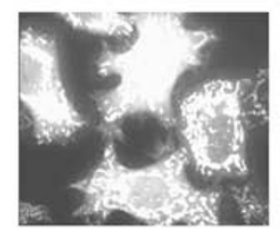

Tim23
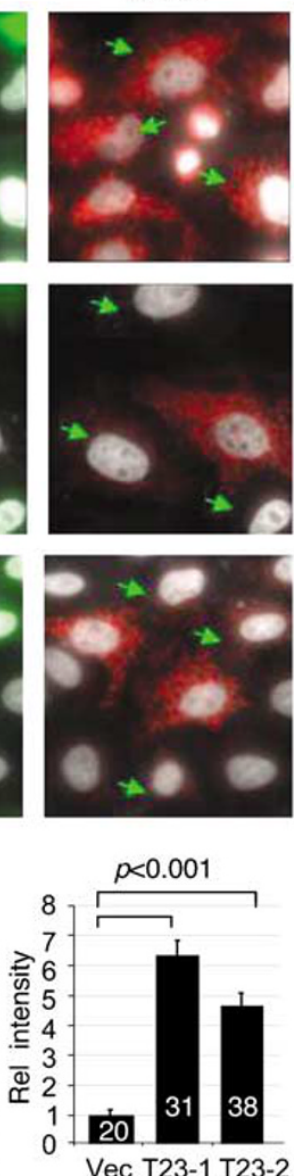

T23-2+mtGFP

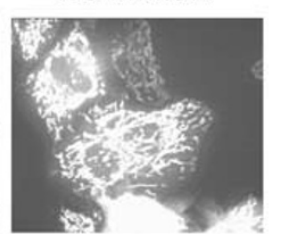

b

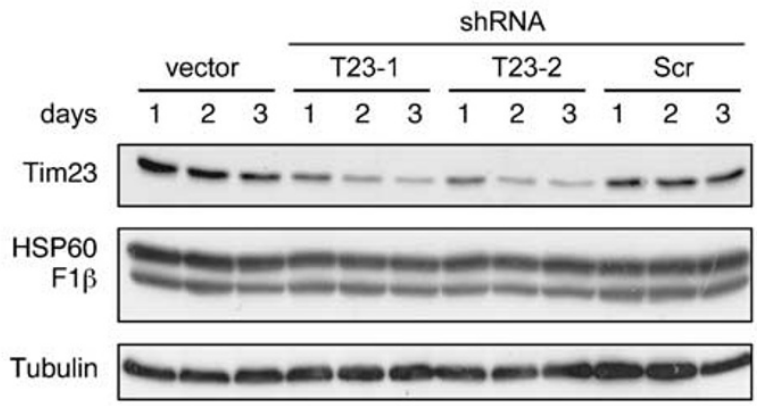

d

$\frac{\text { - Dox }}{\frac{S c r}{s \quad p} \frac{\text { T23-2 }}{s \quad p}} \frac{\text { + Dox }}{\frac{S c r}{s \quad p} \frac{\text { T23-2 }}{s \quad p}}$

mtGFP

29

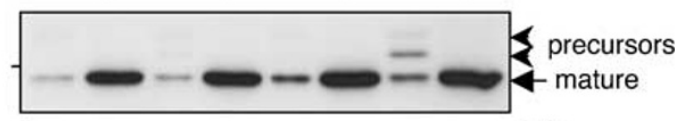

Tim23

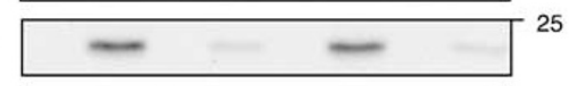

Prohibitin

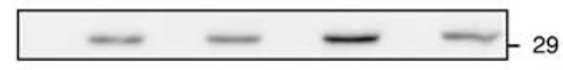

HSP60

Actin
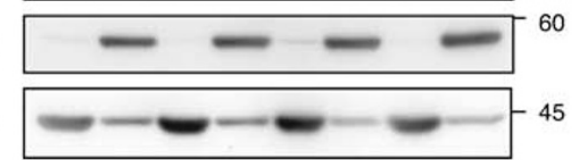

Figure 5 RNAi-mediated knockdown of Tim23 in HeLa cells impairs mitochondrial uptake of matrix-targeted GFP (mtGFP). (a) Cells were co-transfected with EGFP and T23-1 or T23-2 Tim23-specific shRNA expression constructs or the empty cloning vector, and immunostained for Tim23 after 2 days. Nuclei were stained with Hoechst (grey). Arrows show transfected (GFP-positive) cells. Note that Tim23-knockdown cells do not show apoptotic hallmarks, such as blebbing, or nuclear fragmentation and/or condensation. (b) Time course of RNAi-mediated depletion of Tim23 analysed by immunoblotting with antibodies against Tim23, Hsp60, F1 $\beta$, and tubulin (a further loading control). (c) mtGFP and control, or T23-1 or T23-2 shRNA constructs were co-transfected and confocal images were taken after 2 days. Graph shows fluorescence intensity of extra-mitochondrial mtGFP measured in the nucleus (mean \pm S.E.M., number of cells analysed appear inside the bars; data represent one of three independent experiments). (d) Scr or T23-2 shRNA were co-transfected with the doxocylin-inducible pSTAR/mtGFP plasmid. After 2 days, mtGFP expression was enforced in one cohort with $10 \mu \mathrm{g} / \mathrm{ml}$ doxycycline. After $16 \mathrm{~h}$, digitonin-permeabilised cells were fractionated into supernatant (s) and pellet ( $p$ ). Proteins were resolved by PAGE and probed with the indicated antibodies. Arrowheads indicate accumulated pre-processed mtGFP. Results are representative of two independent experiments

cellular function. Significant knockdown of Tim23 protein was obtained by expressing two different shRNA constructs (T231 and T23-2) (Figure 5a and b) while transfection with an empty vector or shRNA containing a scrambled T23-2 targeting sequence (Scr) did not reduce Tim23 expression. The amounts of prohibitin, $\mathrm{F} 1 \beta$, and $\mathrm{Hsp} 60$ were unchanged irrespective of the shRNA constructs (Figure $5 b$ and $d$ ).

Knockdown of Tim23 led to a functional impairment of mitochondrial import of cytosolic proteins into the matrix, detected by following the partition of mitochondrial matrixtargeted GFP (mtGFP) between the cytoplasm and the mitochondria. Thus, HeLa cells in which Tim23 was knocked down retained a statistically significant proportion of diffuse mtGFP in the cytoplasm (Figure 5c), whereas cells expressing an empty shRNA vector (or Scr; data not shown) were devoid of diffuse cytoplasmic fluorescence. The reason mitochondria were still labelled with mtGFP is because the shRNA plasmids and mtGFP were co-transfected to ensure co-expression, but Tim23-knockdown lagged behind the expression of mtGFP. To overcome this problem, and ensure that accumulation of mtGFP in the cytoplasm was due to limited mitochondrial import and not due to leakage of previously imported mtGFP 
out of the mitochondria, we expressed mtGFP from a doxycyline-inducible plasmid. Two days after transfection, mtGFP expression was induced with doxycyline, and after $24 \mathrm{~h}$ the HM pellet and cytosolic fractions were analysed by immunoblotting for the presence of unprocessed mtGFP precursor. Figure 5d shows that Tim23-knockdown cells expressing doxycyline-inducible mtGFP accumulated unprocessed mtGFP precursor (running at higher molecular mass) in the cytosol whereas control cells did not. Incomplete impairment of mitochondrial uptake of mtGFP occurred because expression from the inducible plasmid was leaky despite the absence of doxycyline and thus Tim23-knockdown still lagged behind mtGFP expression. Tim23 depletion in the cell population represented in the blot was also incomplete. Nevertheless, these results show that RNAimediated Tim23 depletion specifically reduces endogenous Tim23 expression sufficiently to impair the matrix import machinery.

We noted that Tim23-depleted cells failed to grow. This was not due to the induction of apoptosis ( $<6 \%$ apoptosis in ScrT23-1-, or T23-2-treated cells 4 days after onset of knockdown; Supplementary Figure S3). By 4 days after Tim23 depletion was induced with shRNA, HeLa cell proliferation was already significantly suppressed by $40 \%$, measured using 5-bromo 2'-deoxyuridine (BrdU) incorporation into nuclear DNA (Figure 6a). BrdU incorporation into mitochondrial DNA appeared unaffected (data not shown). Colony formation was examined after transfection with a puromycinselectable version of the shRNA constructs (pPUR/U6shRNA). Figure $6 b$ shows that although equivalent numbers of cells were present 2 days after the addition of puromycin, no colonies (bar one) were present 14 days after expression of the Tim23-specific shRNAs whereas Scr shRNA expression gave rise to numerous puromycin-resistant clones. Thus, RNAi-mediated loss of Tim23 profoundly impairs cell survival and longevity.

To investigate the underlying mechanism for this deficit, we examined whether mitochondrial respiration was impaired but we found no difference in basal or CCCP-stimulated (uncoupled) respiration rates 3 days after onset of Tim23 depletion compared to those of Scr-transfected cells (Table 1)
Moreover, the intensity of mitotracker orange ${ }^{\mathrm{TM}}$ loading was similar between the two groups of cells (Table 1), indicating that there was no severe loss of $\Delta \Psi \mathrm{m}$. Mitochondria were also not fragmented to a significant extent (Table 1). Moreover, Tim23-knockdown cells were capable of apoptosis, shown in response to TNF and $\mathrm{CHX}$ after 4 days (Supplementary Figure S3b and c). Thus, mitochondria are still functional after Tim23-knockdown.

Tim23-GFP is expressed in mitochondria and delays caspase-independent cell death. To test directly whether lack of Tim23 contributes to cell death in apoptotically stimulated, caspase-inhibited cells we looked for a stable form of Tim23 that would not disappear alongside endogenous Tim23. We found that the amount of exogenous Tim23-GFP expression was comparable to that of endogenous Tim23, but, unlike endogenous Tim23, Tim23-GFP was not degraded upon treatment with Sts or

Table 1 Measurement of mitochondrial functions

\begin{tabular}{lcc}
\hline Function & $\begin{array}{c}\text { Control } \\
(\mathbf{S c r})\end{array}$ & Tim23-depleted \\
\hline $\begin{array}{l}\text { A. Basal respiration }\left(\mathrm{nmol} \mathrm{O} \mathrm{O}_{2} /\right. \\
\left(\mathrm{min} \times 10^{6} \text { cells) }\right.\end{array}$ & $7.2 \pm 1.6$ & $8.1 \pm 2.3$ \\
$\begin{array}{l}\text { B. CCCP-stimulated respiration } \\
\text { C. Membrane potential index }\end{array}$ & $1.8 \pm 0.2$ & $1.9 \pm 0.03$ \\
D. Mitochondrial fragmentation index & $3.3 \pm 0.8$ & $3.2 \pm 1.2$ \\
\hline
\end{tabular}

A. The rate of oxygen consumption of intact $10^{6}$ HeLa cells 3 days after transfection with Scr- or Tim23-shRNA. Respiration of intact cells was measured using a Clark electrode (mean \pm range from two independent experiments) B. $50 \mu \mathrm{g} / \mathrm{ml}$ oligomycin and $10 \mu \mathrm{M}$ CCCP were added for $15 \mathrm{~min}$ before measurement. Data represent the ratio of respiration rate values measured before and after CCCP addition from the same experiments shown in A C. HeLa cells treated as in A were loaded with $1 \mu \mathrm{g} / \mathrm{ml}$ of Mitotracker Orange ${ }^{\mathrm{TM}}$ for $15 \mathrm{~min}$ after which cells were washed and fluorescent images were captured using the UltraView ${ }^{\mathrm{TM}}$ software under identical settings. Equal areas of cytoplasm and nucleus were imaged from 21 (Scr) or 48 (T23-1/T23-2 shRNA) cells using ImageJ software and the ratio of intensity per cell was calculated $(P=0.4$, unpaired $t$-test) $D$. The length and width of each mitochondrion were measured from transmission electron micrographs using a ruler placed orthogonally and the ratios were calculated $(20$ mitochondria from five independent sections of Scr- or T23-shRNA-treated HeLa cells were measured; $P=0.8$, unpaired $t$-test)

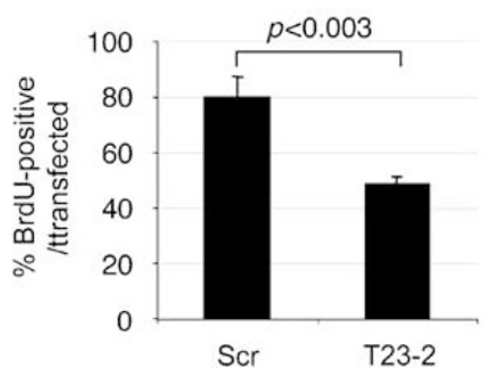

b

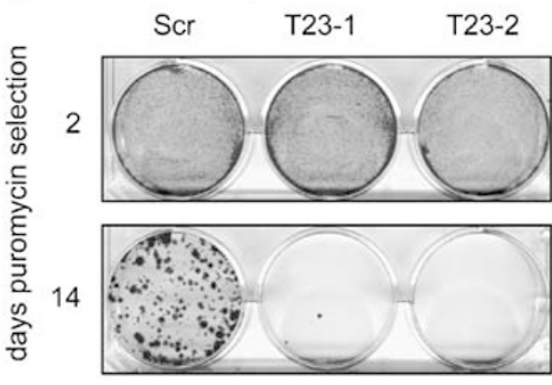

Figure 6 RNAi-mediated knockdown inhibits cell cycling and longevity. (a) HeLa cells were co-transfected with H2B-GFP and the indicated shRNA construct. Cells were incubated with $10 \mu \mathrm{M}$ BrdU (5-bromo 2'-deoxyuridine) between days 4 and 5, then fixed and stained with anti-BrdU. The graph shows percentage of H2B-GFP-positive cells that are also BrdU-positive (mean \pm S.D., three independent experiments; $P<0.001$, unpaired $t$-test). (b) HeLa cells were transfected with the indicated pPUR/U6-shRNA construct. After $6 \mathrm{~h}$, cells were reseeded equally into duplicate wells to which $1 \mu \mathrm{g} / \mathrm{ml}$ puromycin was added after $24 \mathrm{~h}$ to begin selection. Two days later, one set was stained with crystal violet to demonstrate presence of equivalent numbers of transfected, puromycin-resistant cells. The other set was stained after 14 days. Results are representative of three independent experiments 
UV (and BAF) (Figure 7a). Moreover, the majority of Tim23GFP was translocated to mitochondria, although some GFP fluorescence remained in the cytosol compared with mtGFP (Figure 7b). Most notably, expression of Tim23GFP significantly prolonged survival of Sts- and BAF-treated cells after 5 days (Figure 7c and d) compared to cells expressing mtGFP or cytosolic GFP. This preservation was not due to Tim23-GFP-mediated inhibition of apoptosis induction, as it did not prevent apoptosis of Sts-treated HeLa cells in the absence of BAF (Figure 7c). Together, these data show that overexpression of Tim23-GFP, as stable form of Tim23, can counteract the functional consequences of endogenous Tim23 degradation and rescue apoptotically stimulated/caspase-inhibited cells.

\section{Discussion}

Many diseases are associated with mitochondrial dysfunction rendering the host cell prone to death and aging. Some dysfunctions are caused by intrinsic defects such as mtDNA mutations ${ }^{26}$ or mutations in nuclear-encoded mitochondrial proteins, such as the protease paraplegin. ${ }^{27}$ In other cases, mitochondrial dysfunction is triggered from elsewhere in the cell. Our study has revealed a novel mechanism of mitochondrial auto-digestion that proceeds in apoptotically-stimulated cells despite caspase-inhibition. Tim23 degradation is relatively rapid, it being significantly degraded within $15 \mathrm{~h}$ of stimulation with a potent inducer such as Sts, well before COX IV, previously found to be degraded following similar conditions but on a much longer time scale. ${ }^{12,28}$ The relative instability of Tim23 contrasts with the pronounced stability of several other mitochondrial proteins that play key roles in mitochondrial physiology, including inner-membrane proteins that comprise subunits of the various respiratory chain complexes, and prohibitin, thought to stabilise the assembly of the F1-F0 ATPase complex. ${ }^{29}$ Tim23 degradation follows cytochrome $c$ release but does not involve caspases. Indeed, analysis of vertebrate Tim23 sequences (zebra fish to human) failed to locate any obvious caspase cleavage sites.

Tim23 degradation is initiated by MOMP either through a Bax/Bak-dependent event when induced by apoptotic stimuli, or by activating permeability transition in isolated rat liver mitochondria. Why might Tim23 degradation be accelerated by MOMP? One possibility is that Tim23 is destabilised because its association with the IMS protein DPP1/Timm8a is prevented due to the release of DPP1/Timm8a from the mitochondria after MOMP. ${ }^{12}$ Since DPP1/Timm8a facilitates the assembly of Tim23 into the mitochondria under certain conditions, ${ }^{30}$ its absence might prevent proper folding/ insertion of Tim23, leading to abnormal Tim23 topology. Regardless of the cause, we speculate that after MOMP, Tim23 assumes an abnormal conformation within the inner membrane that mitochondrial proteases responsible for quality control detect as 'faulty' and degrade. ${ }^{31}$ We have not yet identified the proteases responsible for proteolysis of Tim23, or the cleavage sites. In preliminary experiments aimed at identifying Tim23-degrading protease(s), we found that the metalloprotease inhibitor 1,10-phenanthroline partially suppressed Atr-induced (but not control) degradation of in vitro-translated $\left[{ }^{35} \mathrm{~S}\right] \mathrm{Tim} 23$ that had been preloaded into rat
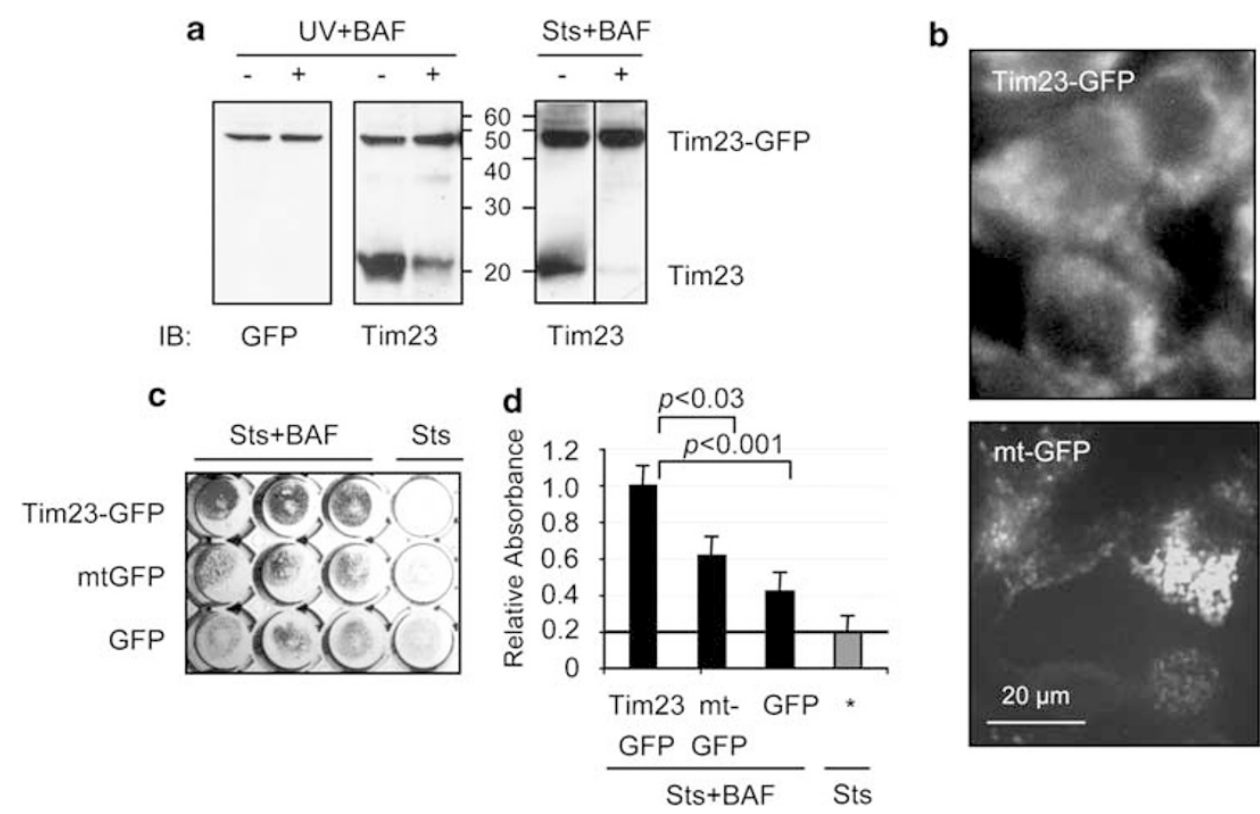

Figure 7 Expression of Tim23-GFP delays caspase-independent cell death. (a) HeLa cells were transfected with Tim23-GFP. After 2 days, cells were left untreated (-) or treated with UV + BAF or Sts + BAF $(+)$. After $24 \mathrm{~h}$, lysates were analysed for expression of Tim23-GFP using anti-GFP or anti-Tim23 antibodies. Note that Tim23-GFP expression is comparable to that of endogenous Tim23; moreover, Tim23-GFP is stable whereas endogenous Tim23 is degraded. Results are representative of 3-4 independent experiments. (b) Fluorescent images of HeLa cells expressing Tim23-GFP or mtGFP. Tim23-GFP is primarily loaded into mitochondria although there is some cytoplasmic Tim23-GFP, and some coloclisation of Tim23-GFP in lysosomes. (c) HeLa cells expressing Tim23-GFP, mtGFP, or GFP (three independent transfections) for $24 \mathrm{~h}$ were treated with Sts + BAF or Sts alone and left to grow for 5 days. Cells were stained with crystal violet. Note significantly higher abundance of cells expressing Tim23-GFP compared to mtGFP or GFP. (d) Quantification of relative absorbance from three independent experiments (two in triplicate, one in duplicate). *, combined average of absorbance of Tim23-GFP-, mtGFP-, and GFP-transfected cells treated with Sts and no BAF. Mean \pm S.D., unpaired $t$-test 
liver mitochondria in a sodium carbonate-resistant form, indicating that it had integrated into the membrane. The serine protease inhibitor dichloroisocoumarin did not prevent this proteolysis (Supplementary Figure S4). However, we were unable to prevent degradation of Tim23 in cells using 1,10-phenanthroline because prolonged exposure was toxic. Whether the target of 1,10-phenanthroline is the AAA ATPase protease family responsible for monitoring protein quality ${ }^{27,31}$ awaits confirmation.

The limited lifespan of cells depleted of Tim23 by RNAi suggested that apoptosis-induced Tim23 loss contributes to the increased morbidity of cells treated with caspase inhibitors. Indeed, we found that expression of a nondegradable form of Tim23 (Tim23-GFP) within mitochondria, prolonged the lifetime of apoptosis-induced, caspase-inhibited cells. The necessity of Tim23 for cell survival and function was indicated previously in yeast, where temperaturesensitive Tim23/mas6-1 mutation disrupts mitochondrial protein import and is lethal at nonpermissive temperatures. ${ }^{32}$ Other components of the mitochondrial protein import machinery have also been implicated in causing cell dysfunction. Knockdown of Tim50, the inner-membrane protein that interacts with Tim23, caused developmental abnormalities in zebra fish. ${ }^{33}$ We noted some instability of Tim50 as well, but this occurred after Tim23 is almost completely degraded. In other studies, brain mitochondria from Alzheimer patients accumulated non-glycosylated amyloid precursor protein that was arrested during transit through TOM40/TIM23 complexes. $^{34}$ This led to mitochondrial respiratory dysfunction and reduced ATP production, indicating the importance of the mitochondrial import machinery in maintaining mitochondrial function. Presumably, such events eventually happen to Tim23-depleted mitochondria as well.

In summary, we have described a novel mechanism of mitochondrial destruction in cells that begins with the loss of Tim23 but which does not apparently involve mitochondrial degradation by autophagy. We cannot exclude the possibility that other mitochondrial proteins are also degraded. However, since degradation of Tim23 is sufficient to cause loss of cell viability, lack of Tim23 is another mechanism of potential significance in caspase-independent cell death. Our data imply that manipulating Tim23 expression may have therapeutic potential. Preservation of Tim23 might postpone caspase-independent cell death in diseases that are characterised by the continuous loss of irreplaceable cells, such as neurons or cardiomyocytes. Conversely, selective knockdown of Tim23 in cancer cells might help counteract the development of resistance against chemotherapy.

\section{Materials and Methods}

Reagents and antibodies. Atr, Sts, Eto, CHX, CsA, Eme, AraC, and BrdU were purchased from Sigma. BAF was from Enzyme Systems Products. Oligonucleotides were synthesised by Sigma-Genosys. Monoclonal anti-Tim23 (BD Biosciences, cat. no. 611222) was used at 1:2000 for blotting and at 1:500 for immunocytochemistry; polyclonal anti-Tim23 C19 (Santa Cruz cat. no. SC-13298) and anti-Tim23 T20 (Santa Cruz cat. no. SC-13297) were used at 1:200; antiTom20 (BD Biosciences, cat. no. 611222) was used at 1:5,000 for blotting and at 1:500 for immunostaining; anti-F1 $\beta$ (BD Biosciences cat. no. 612518) was used at 1:10000; Hsp60 (Sigma cat. no. H4149) was used at 1:500; anti-GFP (BD Biosciences cat. no. 8371) was used at 1:1000; anti-prohibitin (AbCam, cat. no. ab2996) was used at 1:200; anti-COX IV (AbCam cat. no. ab16056) was used at
1: 1000; Tim50 (AbCam cat. no. ab23938) was used at 1: 1000; anti-BrdU (DAKO, MO744) was used at 1:50; and anti- $\alpha$-tubulin (Sigma, T5168) was used at 1:4000. The LC3 antibody was a kind gift from Drs. T Ueno and E Kominami (Juntendo University School of Medicine, Tokyo). HRP- or Cy3-conjugated secondary antibodies were from Jackson Immunoresearch Labs (anti-rabbit, anti-mouse) or from Santa-Cruz (anti-goat); Alexa-488-conjugated antibody was from Invitrogen.

Plasmids, adenoviruses, and shRNA constructs. pEGFP-N1 and pEGFP-mito were from Clontech, and pBOS-Histone2B-GFP (pH2B-GFP) was from BD Biosciences. The mtGFP coding sequence of $p E G F P$-mito was subcloned into $\mathrm{PSTAR}^{35}$ a gift from Dr. Q Zeng (IMCB, Singapore), to yield doxycylineinducible mtGFP. Adenoviruses Ad-Puma and $A d-P u m a \triangle B H 3$ were a gift from Dr. B Vogelstein (HHMI, Baltimore). ${ }^{36}$

shRNA plasmids. For the construction of shRNA-expressing constructs, target sequences against human Tim23 were selected using a web-based programme (http://i.cs.hku.hk/ sirna/software/sirna.php). Self-annealing primers were synthesised, containing a TTCAAGAGA loop sequence between the target sequence and its reverse complement sequence as well as a TTTTT-Pol III termination signal. The oligonucleotide had appropriate overhangs to allow cloning in the Apal and EcoRI sites of pSilencer 1.0 U6 from Ambion. The annealed oligonucleotides inserts had the following sequence (the target sequence is underlined):

T23-1 insert:

5'-ATGACAGGCATGTTGTATATTCAAGAGATATACAACATGCCTGTCATGG

TTTTTT- $3^{\prime}$

3'-CCGGTACTGTCCGTACAACATATAAGTTCTCTATATGTTGTACGGACAG

TACCAAAAAATTAA-5'

T23-2 insert:

5'-CTCTGTCTCCTTATTTAAATTCAAGAGATTTAAATAAGGAGACAGAGGG

TTTTTT-3

3'-CCGGGAGACAGAGGAATAAATTTAAGTTCTCT AAATTTATTCCTCTGTC TCCC AAAAAATTAA-5

Scr insert (contains a scrambled T23-2 targeting sequence):

5'-TTATTCTATTCTCGTCAACTTCAAGAGAGTTGACGAGAATAGAATAACCT

TTTTT-3'

3'-CCGGAATAAGATAAGAGCAGTTGAAGTTCTCTCAACTGCTCTTATCTTAT

TGG AAAAAATTAA-5'

To measure colony formation of puromycin-resistant cells, the annealed shRNA cassettes together with the U6 promoter were subcloned into pPUR (Invitrogen) (forming pPUR/U6).

Tim23 cDNA was synthesised from total RNA isolated from Hela cells by reverse transcription using gene-specific primers. The full-length sequence was amplified by PCR and subcloned into pEGFP-N1 to generate pTim23-GFP (Nhel and Kpnl sites are shown in bold):

FL-up AGTTGGTACCGTGAGTGACTGTTGGAGCAAGG

1-down ATCCGCTAGCTCGCCACCATGGAAGGAGGCGGG

Labelling of mitochondrion-encoded proteins. Mitochondrial translation products in HeLa cells were labelled essentially by following the pulse-chase method described by Chomyn. ${ }^{24}$ After adding $\left[{ }^{35} \mathrm{~S}\right]$ Translabel $^{\mathrm{TM}}$ (ICN) for $1 \mathrm{~h}$ in the presence of $100 \mu \mathrm{g} / \mathrm{ml} \mathrm{eme}$, or $100 \mu \mathrm{g} / \mathrm{ml} \mathrm{CHX}$ (cytoplasmic protein synthesis inhibitors), or $100 \mu \mathrm{g} / \mathrm{ml} \mathrm{CHX}$ and $100 \mu \mathrm{g} / \mathrm{ml} \mathrm{CAP} \mathrm{(mitochondrial} \mathrm{protein} \mathrm{synthesis}$ inhibitor), mitochondria from one cohort of cells were harvested immediately (pulse). The other cohort that had been labelled in presence of $\mathrm{CHX}$ was washed free of $\mathrm{CHX}$ and incubated with the indicated drugs for indicated times. The mitochondria-enriched $10000 \times g$ fraction isolated from cells permeabilised with $0.25 \%$ digitonin (which did not cause mitochondrial disruption) was separated by SDS-PAGE, blotted, stained with Ponceau S, dried, exposed to a Kodak phosphorimager screen for 2 days, and scanned using a STORM ${ }^{\circledR}$ scanner (GE Healthcare).

Transfections. Transfections were performed with Lipofectamine 2000 (Invitrogen) using co-transfected pEGFP-N1 or pH2B-GFP as reporter plasmids, and pEGFP-mito or pSTAR/mtGFP to report on mitochondrial import. Reporter plasmids were used at a $1: 3$ ratio with the co-transfected DNA.

Cell culture and apoptosis. HeLa cells, sympathetic (SCG) neurons, and the various MEF lines were cultured as described. ${ }^{6,11,37}$ Bax/Bak single KO and DKO were the generous gift of SJ Korsmeyer (Dana Farber Institute, Boston, MA, 
USA), while the Atg5 wt and Atg5KO MEFs were a generous gift of Dr. N Mizushima (Tokyo Medical and Dental University, Tokyo, Japan). Apoptosis was measured by nuclear fragmentation, imaged with $1 \mu \mathrm{g} / \mathrm{ml}$ Hoechst 33342 . Necrotic cells permeable to propidium iodide were excluded.

Preparation of mitochondria. HeLa cells were dispersed with a $27 \mathrm{G}$ needle in mannitol buffer ${ }^{38}$ containing Complete $^{\mathrm{TM}}$ (Roche) and spun at $500 \times \mathrm{g}$. $\mathrm{HM}$ and cytosolic fractions were separated from the supernatant by centrifugation a $10000 \times g$. In some cases, HeLa cells were permeabilised with $0.25 \%$ digitionin (which does not cause MOMP) before fractionation. Rat liver mitochondria were isolated in a sucrose buffer, washed and then incubated in K/MOPS buffer containing $5 \mathrm{~mm}$ nitrilotetraacetic acid and respiratory substrates. ${ }^{20}$

Immunoblotting and immunofluorescence. Post-nuclear protein extracts were separated by SDS-PAGE, blotted, and analysed using enhanced chemiluminescence (Amersham). Band intensities were quantified using NIH Image 1.62 software. Immunostaining was conducted in PBS containing $0.3 \%$ Triton X100. Fluorescent images were captured at a fixed exposure with a cooled Astrocam 4100 CCD camera with UltraView LCl software (Perkin Elmer). Images were imported into NIH Image for analysis of intensities within nuclear and cytoplasmic compartments.

BrdU incorporation. HeLa cells were co-transfected with pH2B-GFP plus the indicated shRNA construct and after 4 days BrdU $(10 \mu \mathrm{M})$ was added for $24 \mathrm{~h}$. Cells were fixed with $3 \%$ paraformaldehyde, permeabilised with acetone $\left(-20^{\circ} \mathrm{C}, 10 \mathrm{~min}\right)$, rehydrated, and treated ( $30 \mathrm{~min}$ ) with $2 \mathrm{~N} \mathrm{HCl}$. Neutralised cells were incubated with anti-BrdU and detected with a Cy3-conjugated anti-mouse antibody.

Colony formation. HeLa cells transfected with pPUR/U6-shRNA constructs were reseeded after $6 \mathrm{~h}$ at equal density onto two 6-well plates. Selection was begun the next day using puromycin $(1.75 \mu \mathrm{g} / \mathrm{ml})$. After 2 or 14 days, cells were methanol-fixed and stained with crystal violet.

Statistics. P-values were determined using ANOVA followed by Tukey's HSD or by two-tailed Student's $t$-test.

Acknowledgements. We thank S Chapman and S Murfitt from our teaching labs for sharing their excellent mitochondrial preparations, and Drs. J Yu, B Vogelstein, N Mizushima, T Ueno, E Kominami, Q Zeng, S Korsmeyer, and V Dixit for providing invaluable reagents. We also thank Mar González-Barroso for help with the respiration measurements. This work was supported by Programme grant 064232 from the Wellcome Trust.

1. Dolezal P, Likic V, Tachezy J, Lithgow T. Evolution of the molecular machines for protein import into mitochondria. Science 2006; 313: 314-318.

2. Green DR, Kroemer G. The pathophysiology of mitochondrial cell death. Science 2004; 305: $626-629$

3. Tolkovsky AM, Xue L, Fletcher GC, Borutaite V. Mitochondrial disappearance from cells: a clue to the role of autophagy in programmed cell death and disease? Biochimie 2002; 84: 233-240.

4. Lemasters JJ. Selective mitochondrial autophagy, or mitophagy, as a targeted defense against oxidative stress, mitochondrial dysfunction, and aging. Rejuvenation Res 2005; 8: 3-5.

5. Mokranjac D, Neupert W. Protein import into mitochondria. Biochem Soc Trans 2005; 33 1019-1023.

6. Xue L, Murray JH, Tolkovsky AM. The Ras/phosphatidylinositol 3-kinase and Ras/ERK pathways function as independent survival modules each of which inhibits a distinct apoptotic signaling pathway in sympathetic neurons. J Biol Chem 2000; 275: 8817-8824.

7. Wyttenbach A, Tolkovsky AM. The BH3-only protein Puma is both necessary and sufficient for neuronal apoptosis induced by DNA damage in sympathetic neurons. $J$ Neurochem 2006; 96: 1213-1226.

8. Fletcher GC, Xue L, Passingham SK, Tolkovsky AM. Death commitment point is advanced by axotomy in sympathetic neurons. J Cell Biol 2000; 150: 741-754.

9. Donzeau M, Kaldi K, Adam A, Paschen S, Wanner G, Guiard B et al. Tim23 links the inner and outer mitochondrial membranes. Cell 2000; 101: 401-412.

10. Bauer MF, Gempel K, Reichert AS, Rappold GA, Lichtner P, Gerbitz K-D et al. Genetic and structural characterization of the human mitochondrial inner membrane translocase. $J \mathrm{Mo}$ Biol 1999; 289: 69-82.
11. Wei MC, Zong WX, Cheng EH, Lindsten T, Panoutsakopoulou V, Ross AJ et al. Proapoptotic BAX and BAK: a requisite gateway to mitochondrial dysfunction and death. Science 2001; 292: 727-730.

12. Arnoult D, Rismanchi N, Grodet A, Roberts RG, Seeburg DP, Estaquier J et al. Bax/Bakdependent release of DDP/TIMM8a promotes Drp1-mediated mitochondrial fission and mitoptosis during programmed cell death. Curr Biol 2005; 15: 2112-2118.

13. Shimizu S, Kanaseki T, Mizushima N, Mizuta T, Arakawa-Kobayashi S, Thompson CB et al. Role of Bcl-2 family proteins in a non-apoptotic programmed cell death dependent on autophagy genes. Nat Cell Biol 2004; 6: 1221-1228.

14. Mizushima N, Yamamoto A, Hatano M, Kobayashi Y, Kabeya Y, Suzuki K et al. Dissection of autophagosome formation using Apg5-deficient mouse embryonic stem cells. J Cell Biol 2001; 152: 657-668

15. Kabeya Y, Mizushima N, Ueno T, Yamamoto A, Kirisako T, Noda T et al. LC3, a mammalian homologue of yeast Apg8p, is localized in autophagosome membranes after processing. EMBO J 2000; 19: 5720-5728.

16. Tanida I, Ueno T, Kominami E. LC3 conjugation system in mammalian autophagy. Int $J$ Biochem Cell Biol 2004; 36: 2503-2518.

17. Ricci JE, Munoz-Pinedo C, Fitzgerald P, Bailly-Maitre B, Perkins GA, Yadava N et al Disruption of mitochondrial function during apoptosis is mediated by caspase cleavage of the p75 subunit of complex I of the electron transport chain. Cell 2004; 117: 773-786.

18. Cepero E, King AM, Coffey LM, Perez RG, Boise LH. Caspase- 9 and effector caspases have sequential and distinct effects on mitochondria. Oncogene 2005; 24: 6354-6366.

19. Bruni A, Luciani S, Contessa AR. Inhibition by atractyloside of the binding of adenine nucleotides to rat liver mitochondria. Nature 1964; 201: 1219-1220.

20. Xue L, Borutaite V, Tolkovsky AM. Inhibition of mitochondrial permeability transition and release of cytochrome $c$ by anti-apoptotic nucleoside analogues. Biochem Pharmacol 2002; 64: 441-449.

21. Halestrap AP, Davidson AM. Inhibition of $\mathrm{Ca2}(+)$-induced large-amplitude swelling of liver and heart mitochondria by cyclosporin is probably caused by the inhibitor binding to mitochondrial-matrix peptidyl-prolyl cis-trans isomerase and preventing it interacting with the adenine nucleotide translocase. Biochem J 1990; 268: 153-160.

22. Novgorodov SA, Gudz TI, Jung DW, Brierley GP. The nonspecific inner membrane pore of liver mitochondria: modulation of cyclosporin sensitivity by ADP at carboxyatractylosidesensitive and insensitive sites. Biochem Biophys Res Commun 1991; 180: 33-38.

23. Frank S, Gaume B, Bergmann-Leitner ES, Leitner WW, Robert EG, Catez F et al. The role of dynamin-related protein 1, a mediator of mitochondrial fission, in apoptosis. Dev Cell 2001; 1: 515-525

24. Chomyn A. In vivo labeling and analysis of human mitochondrial translation products. Methods Enzymol 1996; 264: 197-211.

25. Meinecke M, Wagner R, Kovermann P, Guiard B, Mick DU, Hutu DP et al. Tim50 maintains the permeability barrier of the mitochondrial inner membrane. Science 2006; 312: 1523-1526.

26. Krishnan KJ, Greaves LC, Reeve AK, Turnbull DM. Mitochondrial DNA mutations and aging. Ann NY Acad Sci 2007; 1100: 227-240

27. Koppen M, Langer T. Protein degradation within mitochondria: versatile activities of AAA proteases and other peptidases. Crit Rev Biochem Mol Biol 2007; 42: 221-242.

28. Xue L, Fletcher GC, Tolkovsky AM. Mitochondria are selectively eliminated from eukaryotic cells after blockade of caspases during apoptosis. Curr Biol 2001; 11: 361-365.

29. Osman C, Wilmes C, Tatsuta T, Langer T. Prohibitins interact genetically with Atp23, a novel processing peptidase and chaperone for the F1Fo-ATP synthase. Mol Biol Cell 2007 18: $627-635$.

30. Rothbauer U, Hofmann S, Muhlenbein N, Paschen SA, Gerbitz KD, Neupert W et al. Role of the deafness dystonia peptide 1 (DDP1) in import of human Tim23 into the inner membrane of mitochondria. J Biol Chem 2001; 276: 37327-37334.

31. Augustin S, Nolden M, Muller S, Hardt O, Arnold I, Langer T. Characterization of peptides released from mitochondria: evidence for constant proteolysis and peptide efflux. J Biol Chem 2005; 280: 2691-2699.

32. Emtage JL. Jensen RE, MAS6 encodes an essential inner membrane component of the yeast mitochondrial protein import pathway. J Cell Biol 1993; 122: 1003-1012.

33. Guo Y, Cheong N, Zhang Z, De Rose R, Deng Y, Farber SA et al. Tim50, a component of the mitochondrial translocator, regulates mitochondrial integrity and cell death. J Biol Chem 2004; 279: 24813-24825.

34. Devi L, Prabhu BM, Galati DF, Avadhani NG, Anandatheerthavarada HK. Accumulation of amyloid precursor protein in the mitochondrial import channels of human Alzheimer's disease brain is associated with mitochondrial dysfunction. J Neurosci 2006; 26 : 9057-9068

35. Zeng Q, Tan YH, Hong W. A single plasmid vector (pSTAR) mediating efficient tetracyclineinduced gene expression. Anal Biochem 1998; 259: 187-194.

36. Yu J, Zhang L, Hwang PM, Kinzler KW, Vogelstein B. Puma induces the rapid apoptosis of colorectal cancer cells. Mol Cell 2001; 7: 673-682.

37. Kuma A, Hatano M, Matsui M, Yamamoto A, Nakaya $\mathrm{H}$, Yoshimori T et al. The role of autophagy during the early neonatal starvation period. Nature 2004; 432: 1032-1036.

38. Martinou I, Desagher S, Eskes R, Antonsson B, Andre E, Fakan S et al. The release of cytochrome $c$ from mitochondria during apoptosis of NGF-deprived sympathetic neurons is a reversible event. J Cell Biol 1999; 144: 883-889. 